\title{
Smart Rural E-Bus System Using Global Radio (GloRa)
}

\author{
Shu-Hui Lin, ${ }^{1 *}$ Yen-Hui Kuo, ${ }^{1}$ Eric Hsiao-Kuang Wu, ${ }^{1}$ \\ Hou-Sheng Lin, ${ }^{1}$ Emery Jou, ${ }^{2}$ and Ming-Hui Jin ${ }^{3}$ \\ ${ }^{1}$ Dept. of Computer Science and Information Engineering, National Central University, \\ No. 300, Zhongda Rd., Zhongli District, Taoyuan City 320317, Taiwan (R.O.C.) \\ ${ }^{2}$ Information Technology and Management Program of International College, Ming Chuan University, \\ No. 5 De Ming Rd, Gui Shan District, Taoyuan City 333321, Taiwan (R.O.C.) \\ ${ }^{3}$ School of Internet Economics and Business, Fujian University of Technology, \\ No. 3, Xueyuan Rd, University Town, Minhou, Fuzhou City, Fujian Province 350025, China
}

(Received December 31, 2020; accepted March 24, 2021)

Keywords: rural transportation, rural communication, 4G/LTE, LPWAN, cloud computing, fog computing, LoRa, GloRa

In this study, we focus on the electronic bus (E-Bus) system developed for Taiwan's mountainous areas. The rural area in Taiwan with its towering mountains has become a sightseeing hot spot. The radio propagation of a (cloud-based) 4G/LTE network may be severely limited by the mountainous geography, leading to unacceptable connection quality. We have built a smart (fog-based) local wireless E-Bus system using a feature-enhanced long-range widearea network (LoRaWAN, also known as GloRa in Taiwan). Since there is no electricity on the mountain roads, the bus stops of this E-Bus system are operated by a dynamo. Furthermore, we present a virtual direction and position algorithm for multiple E-Bus lines. A novel sensing method using the dynamos is proposed to estimate the feasibility of the E-Bus optimization problem.

\section{Introduction}

This is a new era of smart cities, and all developing nations are focusing on making their cities smart in order to become sustainable and resilient. With the help of Internet of Things (IoT) technologies, a network of physically connected devices enables 'things' to connect and exchange data. IoT creates opportunities to converge the physical and the digital-via data analytics-to improve efficiency, derive benefits, and improve livelihoods. The most important factor determining the success of smart cities is a high-quality digital infrastructure, usually in the form of a cloud-based architecture, to support connectivity among millions of devices.

Smart cities extend the metropolitan area to suburbs such as towns and villages. The E-Bus system is the most commonly used transportation service for travelers and residents in smart cities, towns, and villages. Outside urban areas, there is a much larger suburban area including rural and remote regions. In this study, we aim to improve the E-Bus system developed for Taiwan's mountainous areas, whose towering mountains attract many visitors on a daily basis.

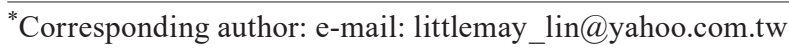
https://doi.org/10.18494/SAM.2021.3288 
Recently, Boshita et al. ${ }^{(1)}$ developed an IoT-based bus location system using a long-range wide-area network (LoRaWAN). A prototype system was evaluated in Nisshin City, Japan. Its bus stops consisted of a LoRa device, a microcomputer, and e-paper as the display medium, powered by a solar panel. With the usage of LoRaWAN $(920 \mathrm{MHz})$, they compressed the time and position information acquired from the GPS to enable it to be effectively transmitted. They confirmed that an IoT-based bus location system can be realized at a lower cost than a bus location system using $4 \mathrm{G} / \mathrm{LTE}$.

An intelligent transportation system on rural roads requires both a sustainable and extendable communication infrastructure to deliver smart and safe services. The rapid growth of low-power wide-area network (LPWAN) technologies has provided a remedy for rural communication. Specifically, when radio propagation of the (cloud-based) 4G/LTE network is severely limited by path loss, shadowing, or a Doppler shift due to the mountainous geography, the connection quality becomes unacceptable. To build a robust rural E-Bus system, a feature-enhanced LoRaWAN (also known as GloRa in Taiwan) will be used to report bus locations in the rural area.

Note that rural areas have a limited power supply due to terrain constraints. ${ }^{(2)}$ In addition to the global energy crisis and the issue of global warming, smart rural area development should support self-generation and zero-emission technology as part of the transition to a green economy. As well as having no electricity, roads in Taiwan's mountainous areas may also lack sunlight due to trees and foggy weather. Instead of a solar panel, we use a dynamo to activate bus stops for radio communication. ${ }^{(3)} \mathrm{A}$ dynamo charger is a hand-cranked power generator for power generation when cycling indoors or outdoors. Since there is no electricity on mountain roads in Taiwan, the bus stops of the rural E-Bus system are powered by generators to receive information on the arrival of buses in a bitwise data transmission format. The rural E-Bus bus stops use energy-saving mini LED lights to display bus locations. Additionally, passengers waiting at the bus stop may crank the generator to obtain bus arrival information.

Owing to unacceptable delays in 4G/LTE services in mountainous terrain, seamless internet connectivity does not exist in rural mountainous regions of Taiwan. Using GloRa for reliable communication and storing the transportation data at rural data centers, the rural E-Bus system can run the bus operation without the internet. The rural E-Bus system creates a low-latency network connection and enables a quick response time. ${ }^{(4)}$ Innovative IoT applications are expected to include not only transportation but also those in the home, health, agriculture, retail, and forestry. Driven by emerging new mobile technologies (such as $5 \mathrm{G}$, fog computing, etc.), new machine-type communication will move the world towards a responsive smart service entity. ${ }^{(5)}$ Responsive machine-type communications are moving IoT scenarios towards a superconnected world. Future rural networks need to be location-aware, energy-efficient, costeffective, sustainable, and extensible for IoT applications. The proposed framework of the rural E-Bus system can be further enhanced in the future with appropriate devices to create a sustainable smart village.

The United Nations' Economic and Social Commission for Asia and the Pacific (ESCAP) ${ }^{(6)}$ is calling for enhanced rural transport connectivity to regional and international transport networks in Asia and the Pacific; Australia's New South Wales state ${ }^{(7)}$ is conducting surveys of 
rural and regional buses and on-demand transport services; and the $\mathrm{UK}^{(8)}$ is considering various practices and prospects and trying to develop a way forward for the future provision of transport services in rural areas.

In this study, we develop a flexible and smart bus sensor network using state-of-the-art wireless technology in rural areas without electrical power with tangible benefits to passengers. The rest of this paper is organized as follows. In Sect. 2, transportation communication in rural Taiwan is addressed. In Sect. 3, current IoT communication technologies are introduced. The deployment and evaluation of the rural E-Bus system are presented in Sect. 4. The fogcomputing-based smart rural IoT platform is discussed in Sect. 5. The paper is concluded in Sect. 6.

\section{Transportation Communication in Rural Taiwan}

Over the last 10 years, Taiwan's central government has initiated several smart-city development projects, and a variety of smart-city applications have been launched through various development strategies and funding. Most have effectively leveraged Taiwan's strengths in ICT technologies, and as a result, many cities in Taiwan have won worldwide recognition and accolades, such as the Intelligent Community of the Year by the Intelligent Community Forum (ICF) (2006 Taipei City, 2013 Taichung City, and 2019 Taoyuan City). In 2014, we proposed an agile urban-parking recommendation service with accurate prediction through the analysis of collected real-time records. ${ }^{(9)}$

However, the most widely used service is the E-Bus system, which provides the real-time locations and arrival information of buses, saving people time and increasing efficiency. Most of Taiwan's local governments have an urban and rural (suburban) development office to provide city planning and social housing services. We deployed a new E-Bus system using a two-way government digital mobile radio and enhanced Wi-Fi (BestLINK) in the rural (suburban) community of PingZhen, Taiwan in 2016. ${ }^{(10)}$

In recent years, 4G/LTE wireless networks have been widely deployed in Taiwan. In this paper, we focus on the E-Bus system for bus services in rural regions. While passengers wait for their bus, they can obtain information on the bus through the intelligent bus stop.

However, the signal reception of existing 4G/LTE network communication services (cloudbased) is intermittently unstable due to the mountainous geography. Cellular network operators often exclude rural areas due to the low average revenue per user and the high cost of infrastructure (macro-cells). ${ }^{(11)}$ This results in poor communication quality in rural areas and makes bus arrival information unreliable and inaccurate. For instance, GPS messages between 15:18 and 15:56 may be delayed until 16:06, GPS messages between 15:56 and 16:04 may be delayed until 16:07, and after 16:11, the GPS messages may be sent and received immediately. As a result, existing traffic information may not be accurate or timely.

After the withdrawal of $2 \mathrm{G}$ communications in Taiwan in 2017, the quality of communications in rural areas became even more unstable. In the winter of 2018, Taiwan's 3G communications was also withdrawn. We must therefore design another system to make transportation communications stable and accessible. 


\section{IoT Communication Technologies}

In recent years, several types of wireless networks have been widely deployed. In this work, we focus on the E-Bus system for movements of buses. Passengers can receive bus information through the intelligent bus stop while they wait for the bus. In Taiwan's current environment, transportation information is provided through specific mobile network operators. ${ }^{(12)}$

Low-power wide-area (LPWA) wireless technology enables IoT applications, which usually require relatively low power consumption and coverage over a relatively long range. Some of the most widely known and deployed LPWA networks include LoRa, NB-IoT, LTE-M, and ultranarrowband. LPWANs enable devices to have a battery life longer than 10 years and require very little expense for low-speed applications. LPWANs have been demonstrated to be an appropriate wireless solution for smart-city scenarios characterized by the sporadic and intermittent transmission of small packets to meet the requirements of long-range, low-power, and low-cost IoT applications. ${ }^{(13)}$

\subsection{G/LTE}

In locations where fixed lines and satellite alone cannot offer the flexibility required to scale a distributed area or deliver a cost-effective solution, 4G/LTE networking can. Offering true flexibility, "things" can be connected in virtually any location at any time using the widespread cellular networks already in place. With the high speeds and bandwidth available over 4G/LTE networks, a cellular solution can connect even the most advanced IoT applications.

The leading cellular networking technologies are mostly cloud-managed with advanced security features and capabilities. This not only enables organizations to ensure the security of their most remote IoT network edges but also ensures that the distributed networks of 4G/LTE routers and gateways are easy and cost-effective to deploy and manage.

\subsection{LoRa vs GloRa}

The LPWAN LoRa technology has been studied by testing and evaluating its range, signalquality properties, and performance in delivering data. ${ }^{(14)}$

In Taiwan, there is a feature-enhanced LoRa technology called GloRa. ${ }^{(15)}$ GloRa is based on Super TaiRa, ${ }^{(16)}$ a collaboration between the National Chung-Shan Institute of Science and Technology (NCSIST) and K-Best Technology, Inc., in Taiwan. It enhances the transmission capability of LoRa. First, the advanced forward error correction (FEC) resolves the high packet error rate (PER) and degradation of sensitivity, which are potential problems of LoRa. Then, an extra high-performance filter (HPF) is added to resolve the problem of poor interference rejection.

A comparison between the features of GloRa and $\mathrm{LoRa}^{(17)}$ is shown in Table 1.

Communication between end-devices and gateways is spread out over different frequency channels and data rates. The traditional LoRa data rates range from 0.3 to $50 \mathrm{kbps}$. The GloRa end-devices may transmit on any channel available at any time, using any available data rate. 
Table 1

GloRa vs LoRa.

\begin{tabular}{|c|c|c|}
\hline Item & GloRa & LoRa \\
\hline Frequency range & 410-990 MHz & $\begin{array}{c}\text { EU } 868433 \mathrm{MHz} \\
\text { US } 915 \mathrm{MHz} \\
\text { AS } 430 \mathrm{MHz} \\
\end{array}$ \\
\hline Modulation & GFSK + CSS & GFSK + CSS \\
\hline Data rate & $\begin{array}{l}\text { DSSS: } 10 \text { bps }-10 \mathrm{kbps} \\
\text { GMSK: } 1-250 \mathrm{kbps}\end{array}$ & $0.3-50 \mathrm{kbps}$ \\
\hline Spreading factor & $6-12$ & $7-12$ \\
\hline Tx power output & Up to $20 \mathrm{dBm}$ & Up to $14 \mathrm{dBm}$ \\
\hline Rx sensitivity & $\begin{array}{c}-118--136 \mathrm{dBm} @ \text { Channel } \\
\text { BW=125 kHz } \\
-115--133 \mathrm{dBm} @ \text { Channel } \\
\text { BW=250 kHz } \\
-112--130 \mathrm{dBm} @ \text { Channel } \\
\text { BW=500 kHz@1\% PER }\end{array}$ & $\begin{array}{c}-123--136 \text { dBm@Channel } \\
\text { BW=125 kHz } \\
-120--133 \text { dBm@Channel } \\
\text { BW=250 kHz } \\
-116--130 \text { dBm@Channel } \\
\text { BW=500 kHz }\end{array}$ \\
\hline Transmission & Up to 100 km@LOS & Up to $20 \mathrm{~km} @ \mathrm{LOS}$ \\
\hline
\end{tabular}

DSSS: Direct Sequence Spread Spectrum

GMSK: Gaussian Minimum Shift Keying

The end-devices change channel in a pseudo-random fashion for every transmission. The resulting frequency diversity of GloRa makes the system more robust to interference.

By reducing the fading loss by $20 \mathrm{~dB}$, the GloRa transmission performance may be 100 times better than that of traditional LoRa. Its built-in military-grade anti-jamming technology and time division multiple access (TDMA) make GloRa suitable for use in Taiwan's rural areas. The deployment and evaluation of the rural E-Bus system will demonstrate and verify the superior properties of GloRa.

\section{Rural E-Bus System}

In the 4G/LTE E-Bus system, vital information such as vehicle location and speed is gathered by the GPS in the vehicle and is transmitted in near real time via cellular or satellite communication to a centralized server maintained in the cloud network. This information is then available for authorized users in real time, and each licensed vehicle owner can access the data at any time in the cloud using a web portal. The development stages and operation procedures are described below.

The 4G/LTE mode of operation is shown in Fig. 1. The bus carries a government-provided traffic recorder GPS system. Through the 4G/LTE networks, the traffic recorder on bus (1) will transmit the E-Bus GPS location to the remote government server (2A). The local rural-city server (2B) will then be able to obtain the E-Bus information (bus registration, route, etc.) and GPS location from the remote government server. Through GloRa, the local rural-city server will periodically broadcast (every $8 \mathrm{~s}$ ) the E-Bus information and GPS location to E-Bus stop (3). The E-Bus location and schedule will be displayed on the LED display so that passengers waiting at the E-Bus stop can see when the next bus will arrive. 


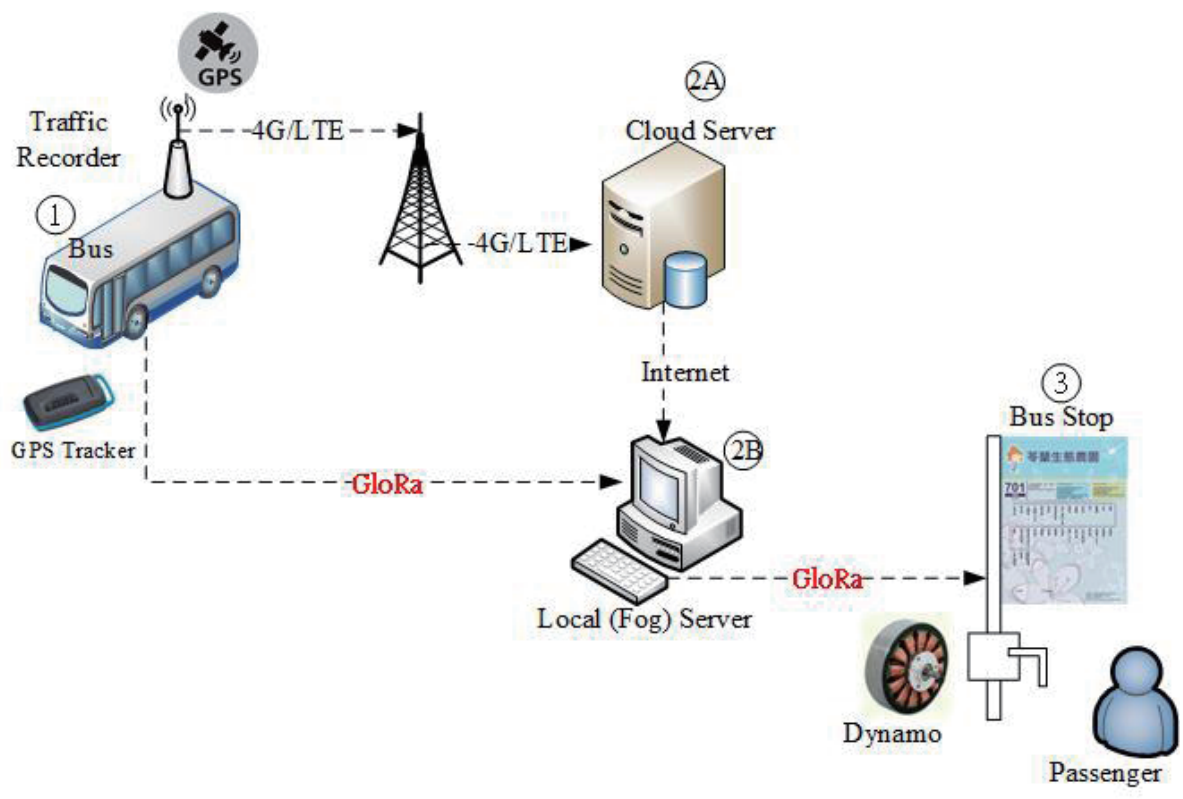

Fig. 1. (Color online) Flow of E-Bus system.

The GloRa mode of operation is also shown in Fig. 1. When 4G/LTE networks are blocked by the mountainous terrain, the government traffic recorder on bus (1) is unable to transmit the E-Bus GPS location to the remote government server. A GloRa GPS tracker (2B) will be used on the bus to transmit the E-Bus GPS location to the local rural-city server (2A). The local E-Bus information may be preloaded from the remote government server. The local rural server will calculate the E-Bus location and schedule and broadcast them to bus stop (3) equipped with a GloRa module. When a passenger cranks the dynamo, the bus stop containing the GloRa module will be activated and the LED display will be charged. The E-Bus location and schedule will be displayed on the LED display. Passengers waiting at the E-Bus stop can see when the next bus will arrive. Unlike in previous studies, a downlink will be used to send data to the end-device (bus stop). In addition, this study also adds complexity. A base station (gateway) sends information on three different routes $(109,110$, and 111) to eight bus stops at the same time. These eight bus stops only receive their own route information. (A bus stop shows information only from one route.) The gateway uses TDMA to send data in order.

Figure 2 shows the hardware interior and the printed circuit board (PCB) of the GloRa gateway. In this study, the rural E-Bus system has been designed with GloRa to allow long-range communications at a low bit rate and low power consumption to serve mountainous rural areas within more than $20 \mathrm{~km}$ using a single base station. The rural E-Bus stop can display bus locations using power generated by the dynamo. When a passenger cranks the $6 \mathrm{~W}$ mini power generator for less than $10 \mathrm{~s}$, the bus stop begins receiving data from the gateway to be displayed on the LED (shown in Fig. 3). The dynamo not only generates power for the bus stop but also senses the number of passengers near the bus stop by recording the hand-cranking events. In most cases, the frequency of the hand-cranking events increases with the number of passengers and the wait time. We also plan to optimize E-Bus schedules according to a passenger- 


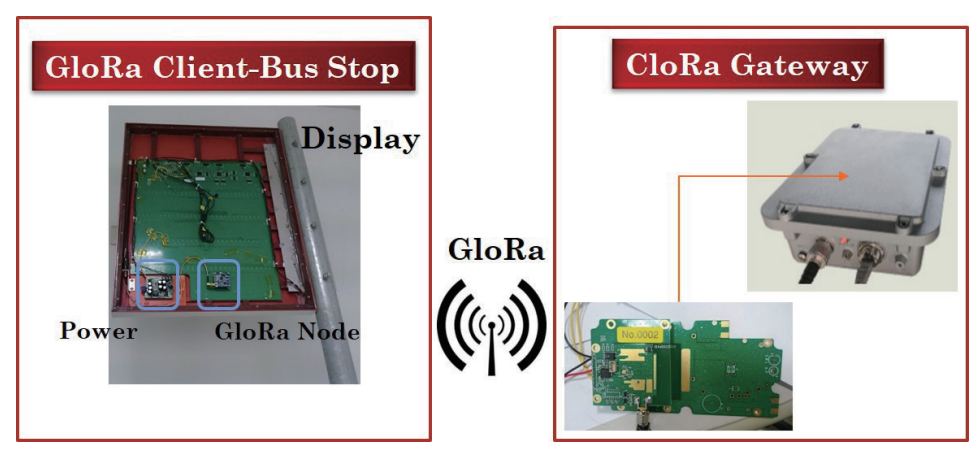

Fig. 2. (Color online) Hardware of the E-Bus stop.
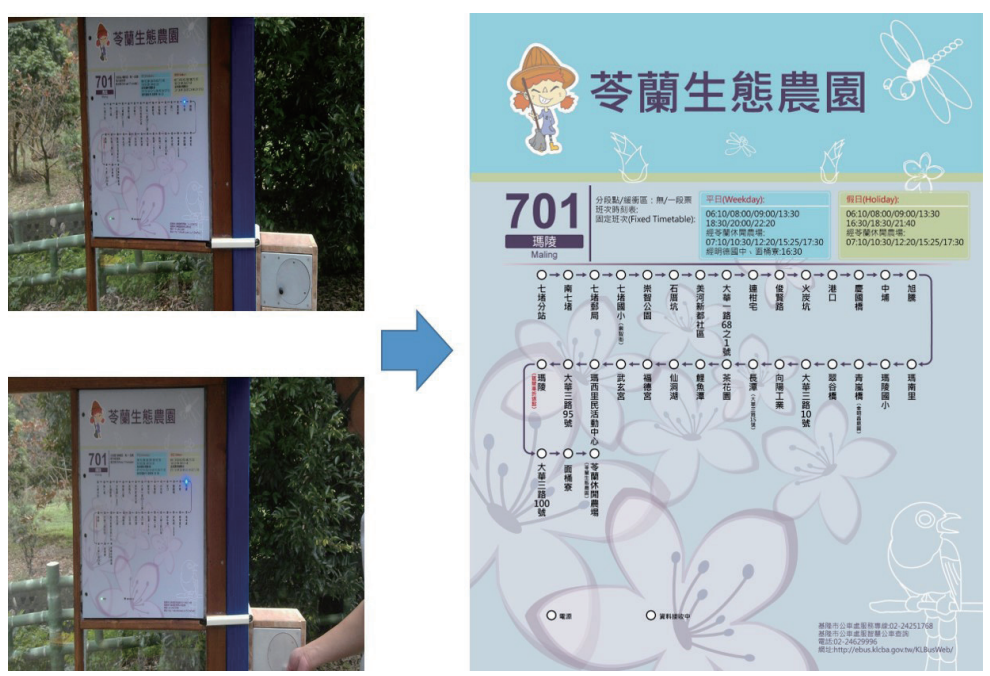

Fig. 3. (Color online) Rural E-Bus stop.

information-gathering model estimated from the hand-cranking events. Optimization of the E-Bus schedule will aim to minimize the wait time of passengers by adjusting the departure time of the E-Bus. We briefly introduce the proposed sensing method for E-Bus optimization in Sect. 4.5.

The rural E-Bus system overcomes the problems of mountainous rural areas where there is a lack of both wireless network (4G/LTE) coverage and power energy infrastructure. The system provides bus information services in mountainous rural areas as urban areas do. It realizes equity, simplicity, and green services in mountainous, rural, and aboriginal areas. The rural E-Bus system with GloRa has already been deployed in both Hsinchu County and Keelung City of Taiwan for bus transportation services.

\subsection{Deployment}

A smart transportation system with the rural E-Bus stop was successfully deployed in Jianshih Township, Hsinchu County, Taiwan, on December 8, 2017. The bus route in Jianshih Township is a circular road. The GloRa base station is on the top floor of the Jianshih Township 
Office. The distance to the farthest bus stop, Digital Sky Restaurant, located on the top of a mountain, is $6.14 \mathrm{~km}$.

Figure 4 shows a Google aerial photo of the E-Bus route in Jianshih Township. After its successful deployment in Jianshih Township, we extended the smart transportation system with the rural E-Bus stop service deeper into the mountainous area of Fuxing District, Taoyuan City (see Fig. 5).

The E-Bus route in Fuxing Township, a mountainous indigenous district in eastern Taoyuan City, is a straight uphill and downhill road. Its distance is $16.35 \mathrm{~km}$. The base station of GloRa is at the Ren'ai Pingmian Parking office. The distance to the farthest bus stop, Shalunzi Bridge, is $16.35 \mathrm{~km}$.

The integration of multiple E-Bus systems in various rural areas with the government's centralized bus-control center and a fog-computing-based architecture will be discussed in Sect. 5.

\subsection{Evaluation}

In Taiwan, the 4G/LTE frequency bands are 700,800 , and $1800 \mathrm{MHz}$, and the GloRa in this study uses $433 \mathrm{MHz}$. The transmission power of the 4G/LTE base station is 3.5-9 W, whereas that of GloRa is $5 \mathrm{~W}$. The coverage rate of $4 \mathrm{G} / \mathrm{LTE}$ in Taiwan has reached $89 \%$. However, the actual test found that the average delay time in Jianshih Township and Fuxing District exceeded $20 \mathrm{~min}$.

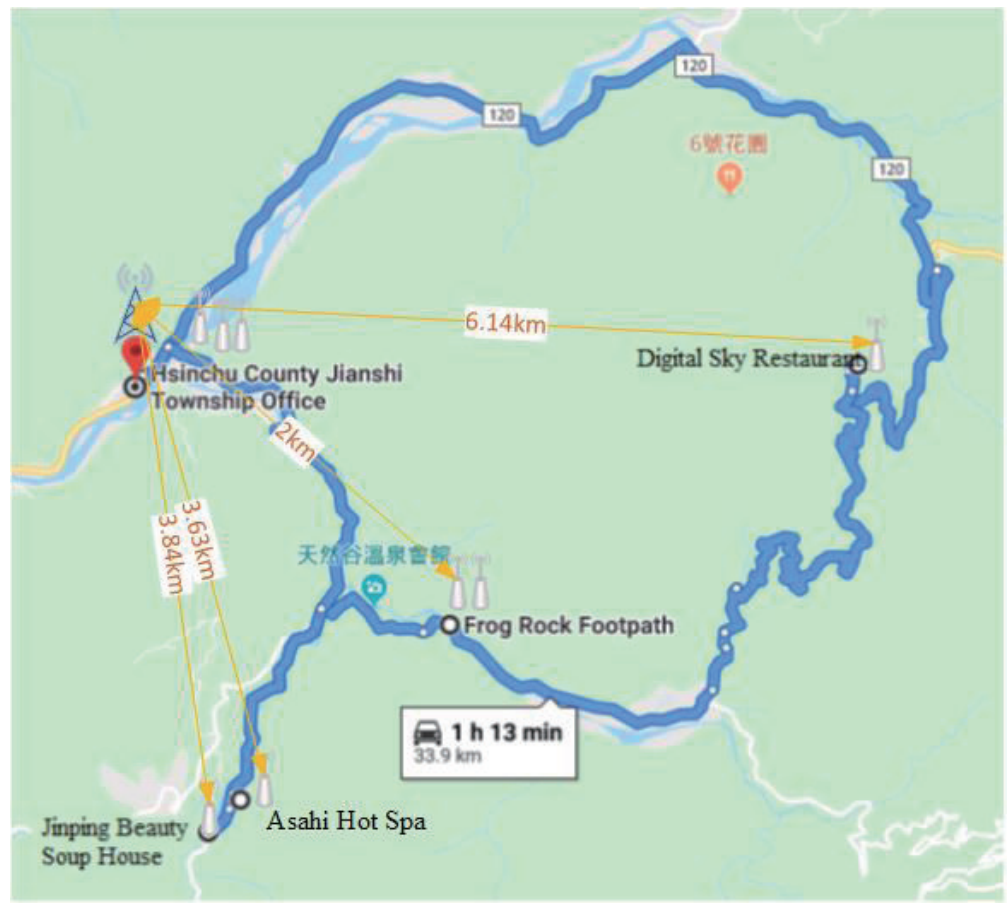

Fig. 4. (Color online) Jianshih District, Shinchu County. 


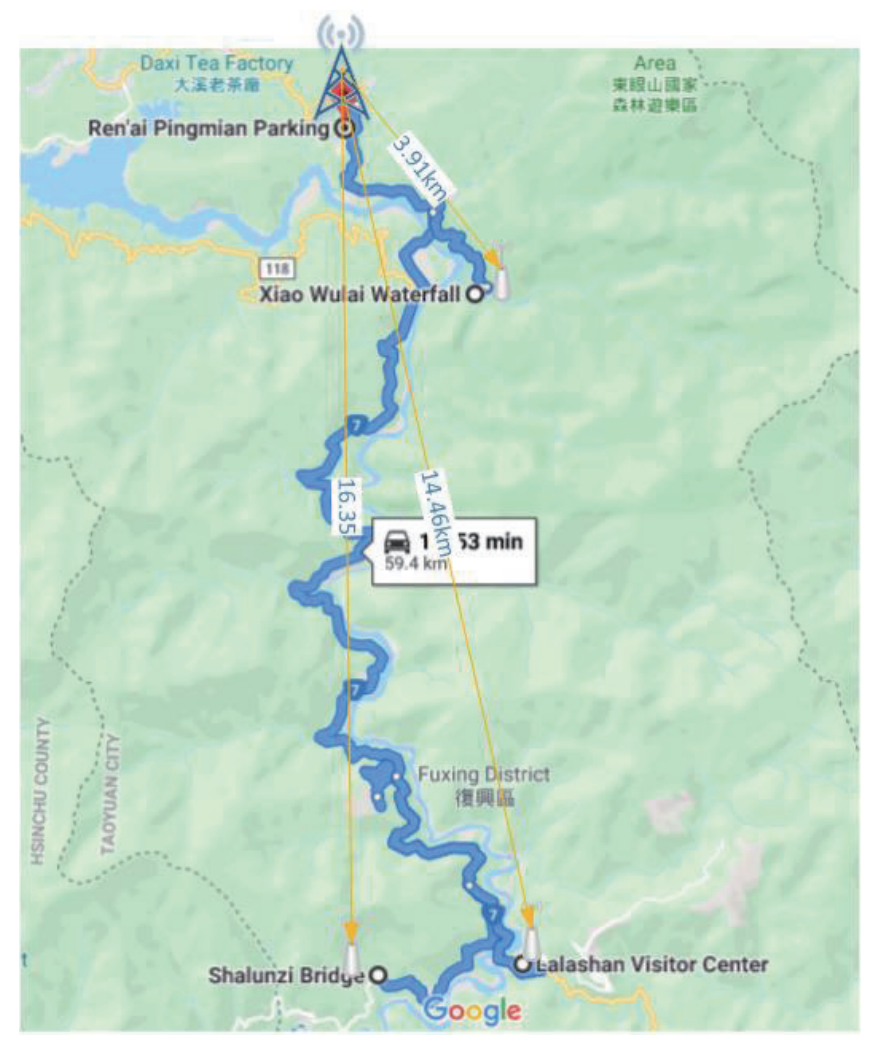

Fig. 5. (Color online) Fuxing District, Taoyuan City.

Before the deployment of GloRa at Jianshih Township and Fuxing District, the rural E-Bus routes were surveyed by the GloRa GPS tracker. A message was sent every $15 \mathrm{~s}$. The RSSI values at Jianshih Township and Fuxing District were between -49 and $-120 \mathrm{dbm}$, within the range of the GloRa receiver sensitivity.

As shown in Fig. 6, at the Lalashan Visitor Center, the RSSI is $-100.2 \mathrm{dbm}$, and at Shalunzi Bridge, it is $-99.4 \mathrm{dbm}$. The distance between these two locations is over $14 \mathrm{~km}$, but the sensitivities are good and the PER is lower than $5 \%$. The communication coverage is thus very good.

\subsection{Data transmission format}

In addition to using bandwidth, data redundancy increases the transmission time at a fixed transmission rate and therefore increases power consumption. Although the data center provides a large amount of bus forecast data, the bus stop only needs to know which stop signs are currently close to the bus. We designed a data format that reduces the information before the bus stop node broadcasts the data to the bus stop.

The data format of the bus information sent to the bus stop is shown in Fig. 7. The information sent determines what the LED must display. There is a size limit for data being transmitted at one time in GloRa (the same as that in LoRaWAN). ${ }^{(17)}$ We propose an 8-byte data transmission 


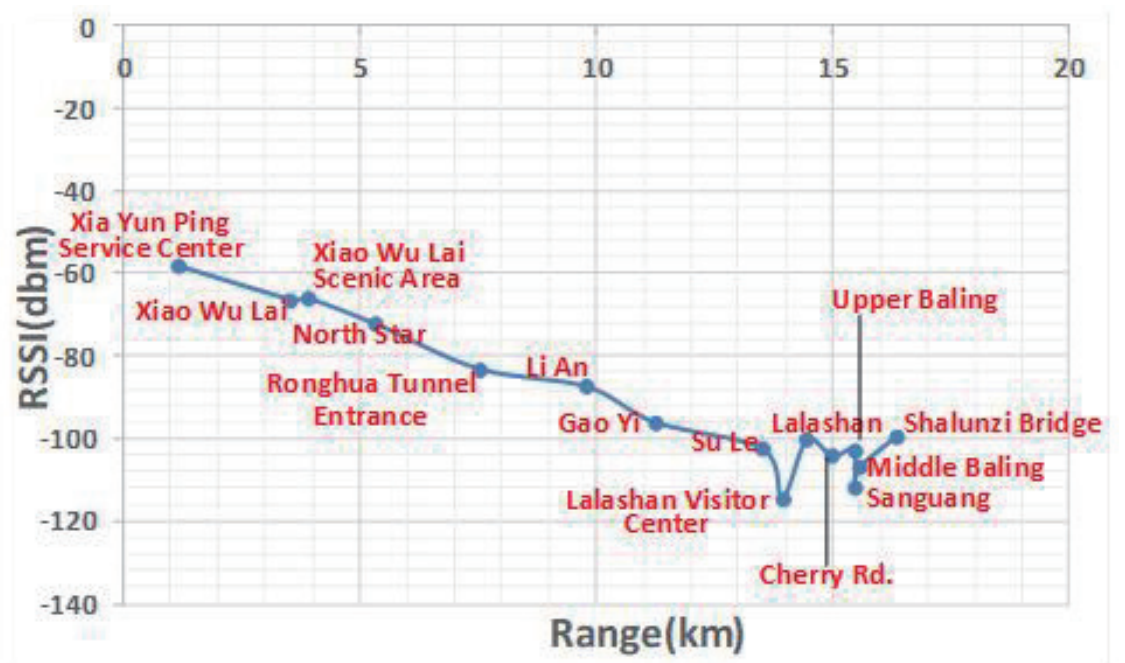

Fig. 6. (Color online) RSSI curve.

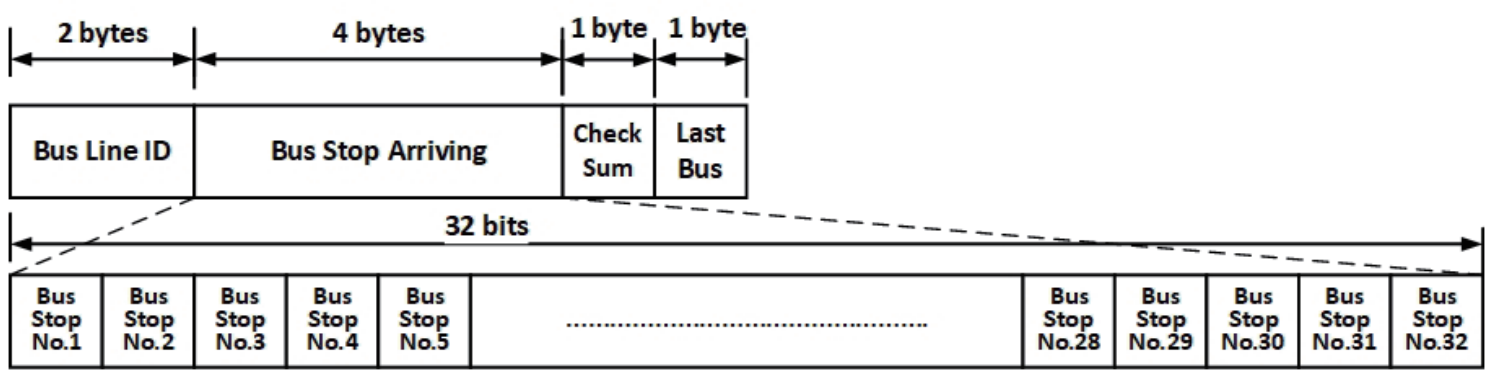

Fig. 7. Data format of bus arrival information.

format for the rural E-Bus stop. Byte 0 and Byte 1 are the bus line ID. Byte 2 through Byte 5 are bus stop locations. One location corresponds to one bit, in which the bit set to one represents the location at which the bus is going to arrive. Thus, four bytes can support a maximum of 32 bus stops on one bus line. Byte 6 is the checksum. Byte 7 indicates whether the bus is the last bus. When the bus stop receives the information, it will light up the LED to show the bus's current position.

In the 4G/LTE data transmission, 230 bytes (JSON format) are used for the arrival data of each stop. If there are $n$ stop locations on one route, $230 \times n$ bytes will be required. Our bitcompressed format will only require eight bytes for a route of 32 bus locations.

\subsection{Virtual direction and position}

We can retrieve the bus's route and other related information from the remote government cloud server and store them at the local rural server, then compute the bus's travel direction and its position. An alternative method is to generate a spline curve (Fig. 8), connecting bus stops as a virtual road, to determine the next correct stop, i.e., the bus direction and its position. 


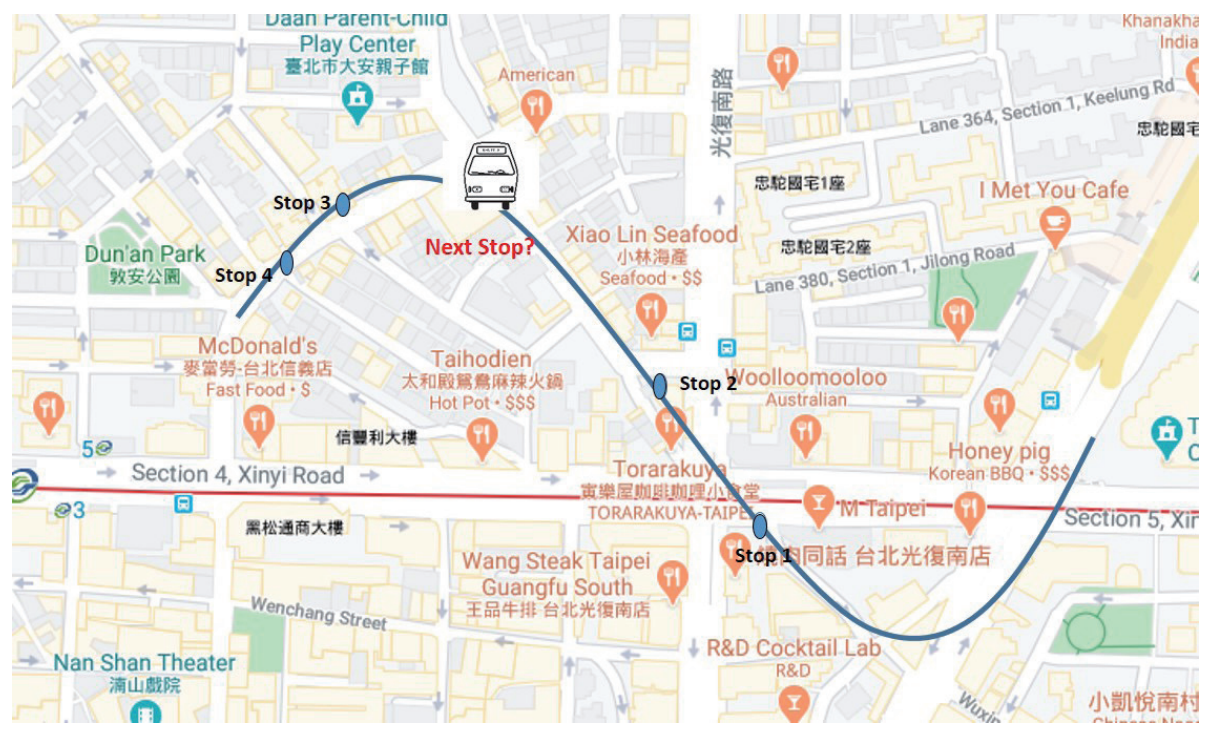

Fig. 8. (Color online) Spline curve of a virtual road.

The effective management of vehicle location information plays a key role in smart rural transportation systems. Using the GPS on the bus, we can retrieve the location information at any time. In addition to the longitude and latitude, we can also the view route ID, direction, time, bus stop sequence, and more. The network must be in good working order for this to be possible. When 4G/LTE does not work, the GPS information will be saved on a local server by GloRa. In this section, we discuss our development of an algorithm to determine the bus's travel direction and its position when only the bus's GPS information is available.

A bus may pass the same location more than once in different directions. Figure 9 shows a bus that travels to Point A twice. When the bus is going east at Point A, its GPS information alone cannot determine whether the bus will continue straight toward B or turn right toward C. However, after inserting virtual bus stops along the route, we can accurately determine the bus's direction of travel.

Note that the next bus stop to Point A may be further than the nearest bus stop in distance. We can see that bus stop C is further away than bus stop B; to which bus stop will the bus travel?

By recording the time and speed of the bus traveling to the bus stop, we can estimate the position of the bus along the route. Figure 9 shows that there are many additional virtual dots inserted between two bus stops. Black dots are bus stops, and white dots are inserted virtual bus stops. When the bus travels to the same location again, we can determine its direction of travel.

Figure 10 shows the algorithm used to build the virtual path.

Build Virtual Path: This function reads the route table from the database, which contains all the stops for a bus route. Route_stop_table includes route_id, stop_id, stop_name, longitude, latitude, and direction, and stores them in a virtual path array to map the bus's current position. Get GPS Data: The bus on-board unit transmits bus position data to the server periodically via GloRa communication. The server then reads the bus position data from the serial port connected to the GloRa base station through RS232. The main data gathered from the bus position are tracker ID, longitude, latitude, direction, and time. 


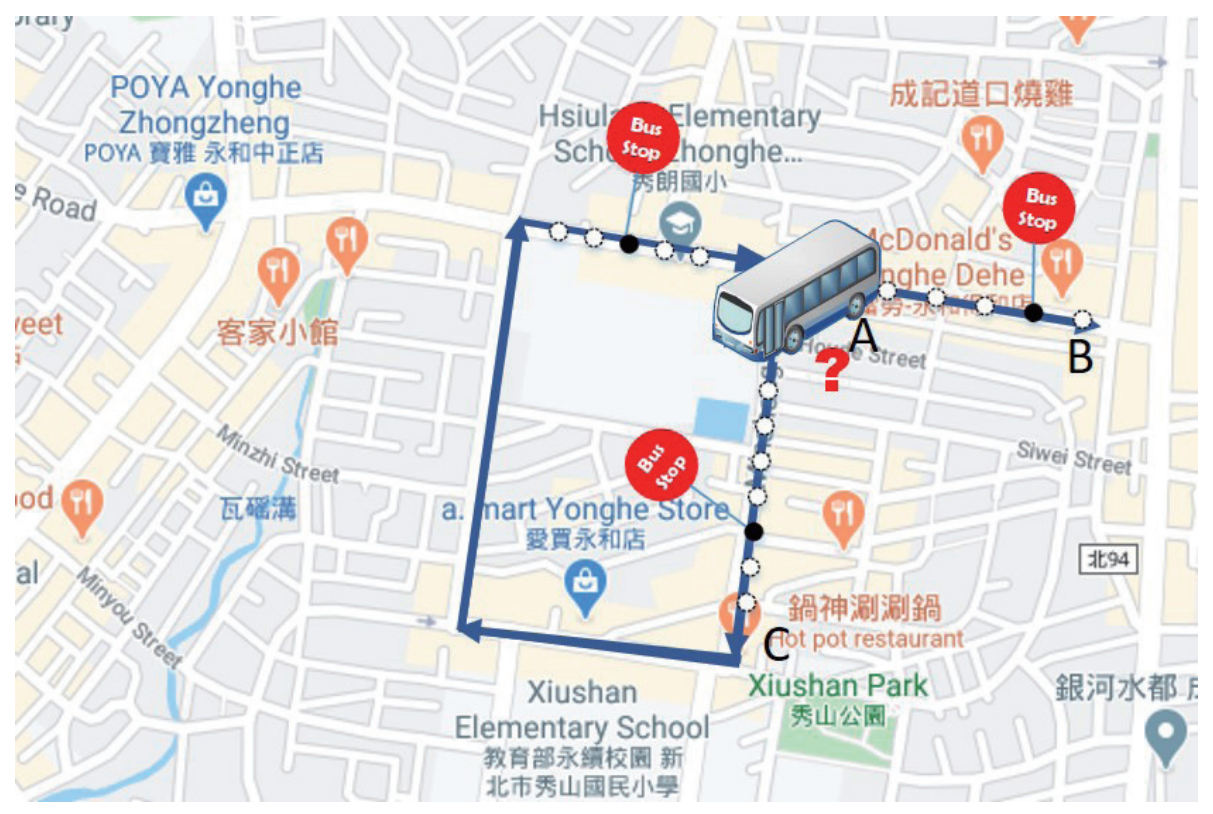

Fig. 9. (Color online) Interpolations of a bus route.

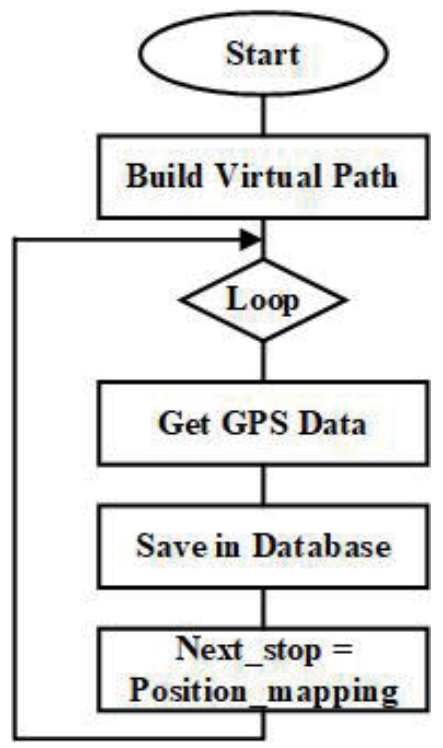

Fig. 10. Algorithm used to build virtual path.

Save in Database: After the bus position data are received, they are saved in the database for bus position mapping.

Position Mapping: This function is based on the GPS longitude, latitude, and direction, which are used as parameters to deduce which bus stop is nearby and to also determine in which direction the bus is headed. When the bus stop ID and the bus direction are confirmed, the function will then return the bus stop ID. 
A stop name with a number is a virtual stop, and a stop number with a name alone is a real stop, i.e., Ziyun Temple 0, Ziyun Temple 1, and Ziyun Temple 2 are virtual stops and Ziyun Temple is a real stop (see Table 2). Because of the virtual bus stops, this bus route is called a virtual route. A station name with a number is usually at a major turn, which is used to identify where the bus is going. In addition, the direction is used to check the direction of the bus, because the same GPS has the directions of forward and return.

\subsection{Proposed E-Bus schedule optimization}

In rural areas, passengers must usually wait a long time for a bus. Bus services are limited due to economic considerations, making it difficult to reduce wait times by simply increasing bus services. With this constraint, in this study, we try to reduce wait times by adjusting the bus departure time schedules.

When the departure time of a bus is provided, its arrival time at each bus stop can be accurately determined. If the E-Bus system proposed in this study learns the passenger-gathering model of each bus stop, then it should be able to estimate wait times if the bus departure schedules are provided. This suggests the possibility of minimizing wait times through employing an appropriate algorithm to determine bus departure schedules.

Learning the passenger-gathering model of each bus stop is the key challenge of minimizing wait times through bus schedule adjustment. Given a bus stop $b$, the passenger-gathering model of bus stop $b$ is a random variable $\Gamma b$ of time. For each time $t, \Gamma b(t)$ is the number of passengers waiting at bus stop $b$.

Although image recognition technologies are helpful in estimating the number of waiting passengers, their power consumption and the cost of installing and maintaining video cameras in rural areas make them unfeasible, forcing us to consider other sensing technologies. In this study, we adopt a dynamo as the sensor on the basis of two assumptions:

Table 2

\begin{tabular}{rccccccc}
\multicolumn{7}{l}{ Virtual route. } & \multicolumn{7}{l}{$l$} \\
\hline $\mathbf{i d}$ & stop_id & stop_name & latitude & longitude & duration & direction & stopindex \\
\hline $\mathbf{0}$ & 0 & Pingzhen City Office & 24.94559 & 121.2183 & 0 & 270 & 0 \\
$\mathbf{1}$ & 1 & Ziyun Temple 0 & 24.94554 & 121.2173 & 24 & 270 & 1 \\
$\mathbf{2}$ & 1 & Ziyun Temple 1 & 24.94549 & 121.2171 & 5 & 180 & 2 \\
$\mathbf{3}$ & 1 & Ziyun Temple 2 & 24.94541 & 121.2178 & 16 & 90 & 3 \\
$\mathbf{4}$ & 1 & Ziyun Temple & 24.94565 & 121.2214 & 87 & 90 & 4 \\
$\mathbf{5}$ & 2 & Beishi Police Station 0 & 24.94563 & 121.2221 & 17 & 90 & 5 \\
$\mathbf{6}$ & 2 & Beishi Police Station 1 & 24.94497 & 121.2220 & 18 & 190 & 6 \\
$\mathbf{7}$ & 2 & Beishi Police Station 2 & 24.94479 & 121.2196 & 59 & 270 & 7 \\
$\mathbf{8}$ & 2 & Beishi Police Station 3 & 24.94262 & 121.2197 & 58 & 180 & 8 \\
$\mathbf{9}$ & 2 & Beishi Police Station & 24.94215 & 121.2206 & 25 & 90 & 9 \\
$\mathbf{1 0}$ & 3 & Zhenping Street 0 & 24.94212 & 121.2208 & 6 & 90 & 10 \\
$\mathbf{1 1}$ & 3 & Zhenping Street 1 & 24.94248 & 121.2228 & 49 & 80 & 11 \\
$\mathbf{1 2}$ & 3 & Zhenping Street & 24.94196 & 121.2230 & 15 & 180 & 12 \\
$\mathbf{1 4}$ & 4 & City CRV 1 & 24.94041 & 121.2220 & 27 & 260 & 14 \\
\hline
\end{tabular}


$\diamond$ The frequency of use of the dynamo increases with the number of passengers waiting at the bus stop.

$\diamond$ The frequency of use of the dynamo increases with increasing wait time at the bus stop.

Figure 11 shows the relationship between the frequency of use of the dynamo and the number of passengers waiting at a bus stop in the $10 \mathrm{~min}$ before a bus arrives. Whenever a bus arrives at a bus stop, the system in the bus counts the passengers who board, and hence the E-Bus system can learn from the data in Fig. 11. Note that the frequency plotted on the vertical axis of Fig. 11 is a random variable.

The results in Fig. 11 support the above two assumptions. On the basis of these assumptions, we assume that minimizing the wait time should be equivalent to minimizing the number of times the bus stop's dynamo is used (cranked).

The focus of future work will be to meet the following challenges:

$\diamond$ Applying image recognition technologies to establish the relationship between the number of uses of the dynamo and the passenger-gathering model.

$\diamond$ Applying the number of uses of each bus stop's dynamo to design appropriate algorithms that minimize wait times and to evaluate the proposed algorithms.

\section{Fog-Computing-Based Smart Rural IoT Platform}

At present, the E-Bus system in smart cities mostly relies on a cloud-based architecture. When there are more buses, the load on the cloud is heavier. Also, the farther the bus is from the cloud, the greater the delay. If a fog architecture is adopted, more nodes can be added and the load on the cloud will be dispersed. The nodes are closer to the cloud than the edge devices, which can reduce the computational delay of the cloud. ${ }^{(18)}$

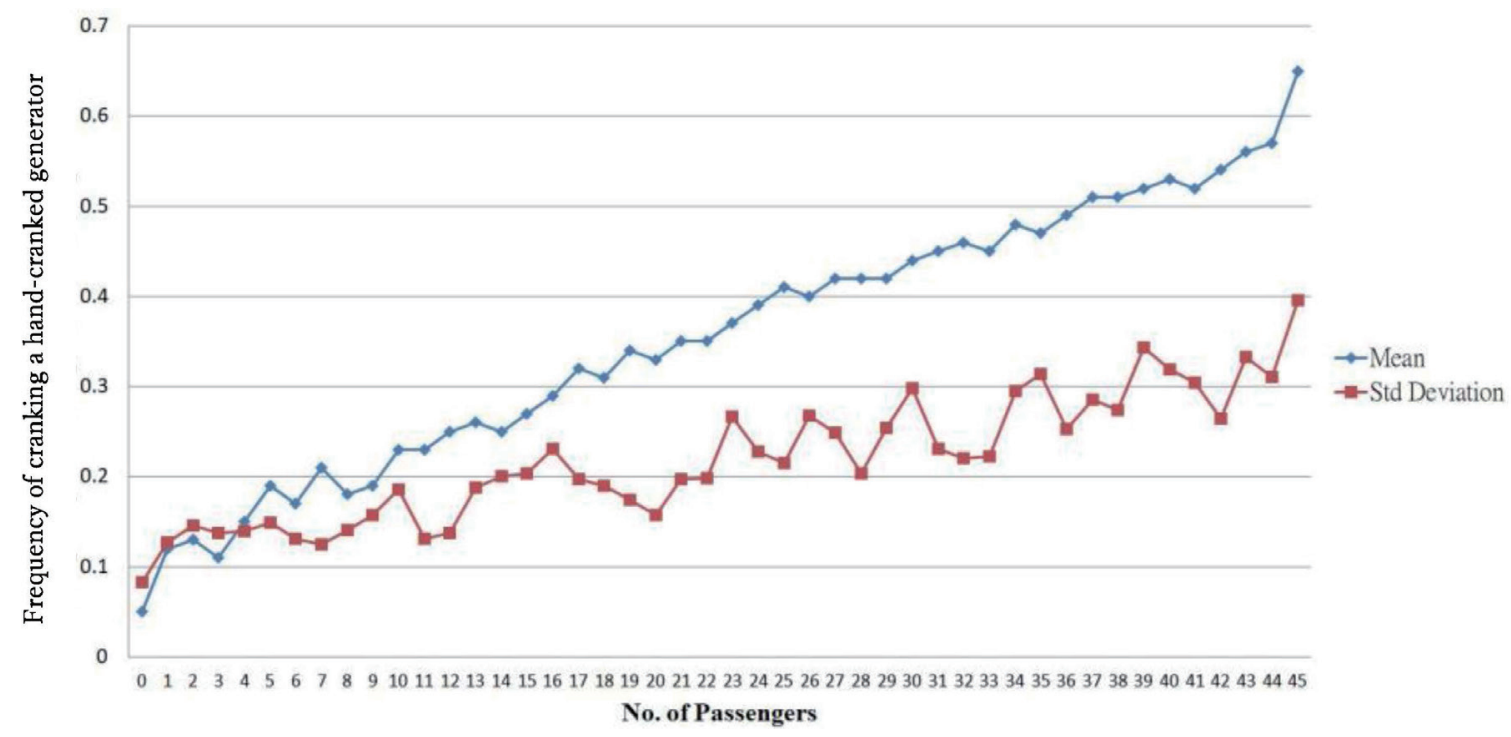

Fig. 11. (Color online) Frequency of dynamo use plotted against number of passengers. 


\subsection{System architecture}

In developing the system architecture, we considered the fragility of external communication systems in rural areas and decided to use a fog computing architecture. Fog computing, or fog networking, is an architecture that uses edge devices to carry out a substantial amount of computation, storage, and communication locally and is routed over the internet backbone. Fog computing consists of edge nodes directly performing physical input and output, often to control a sensor input, display output, or full closed-loop process. The OpenFog consortium pointed out that when a central cloud is unavailable in a particular geography, fog computing can be deployed. However, instead of replacing the cloud-computing model, the fog-computing model serves as an extension of the cloud, working as a distributed decentralized computing infrastructure in which data computing, storage, and applications are distributed in the most logical and efficient way between the data source and the cloud.(19) Service reliability is improved by decentralizing computing resources and communication routing backup mechanisms. The architecture is mainly divided into edge devices, fog nodes, and the cloud.

The E-Bus system will have three layers: cloud, fog, and edge layers (Fig. 12).

Cloud Layer: The data center is in the cloud layer and includes the central manager and bus info database. The central manager is managed by an ITS department of government. The central manager is responsible for instantly providing the bus location and estimating the on-site information storage service. After the local manager calculates the data, the bus data will be stored in the database for inquiry.

Fog Layer: There are bus stop nodes in the fog layer that include the bus info recorder, display unit, and local manager.

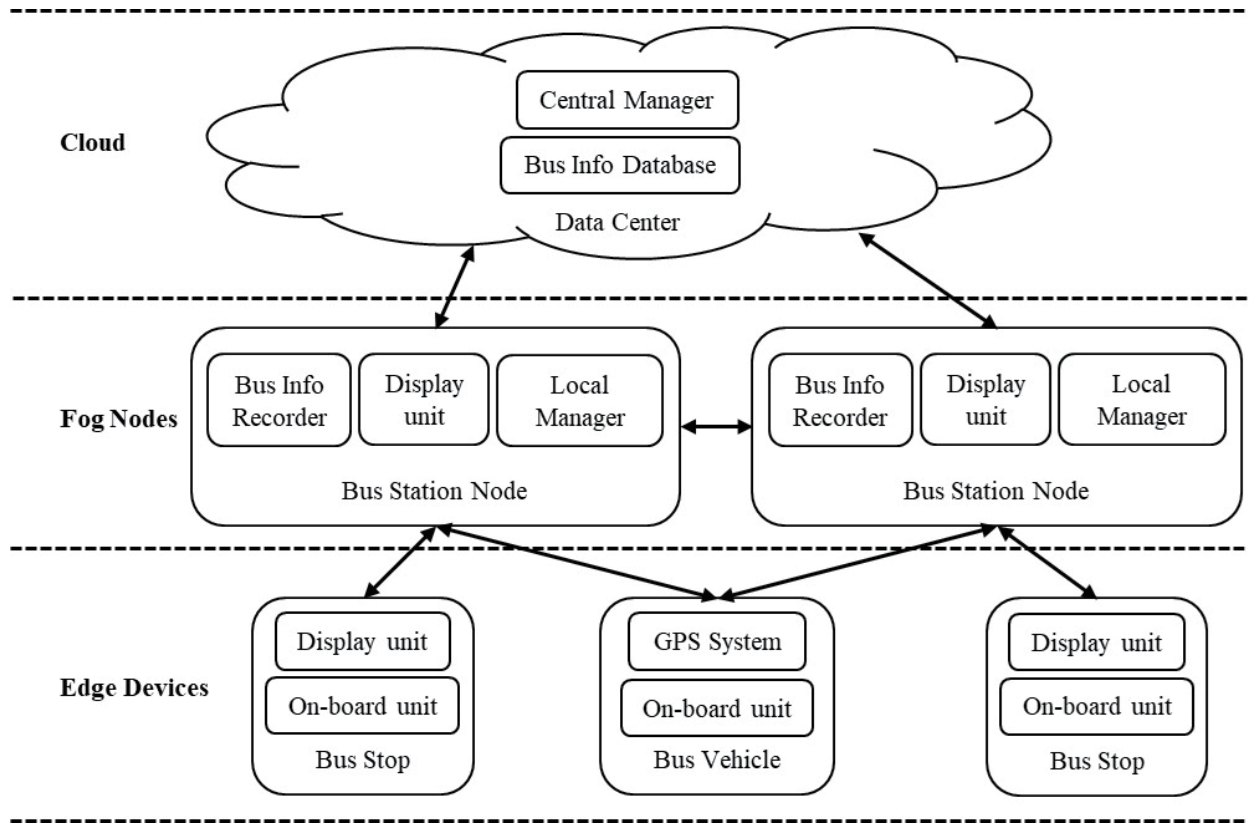

Fig. 12. Model of the E-Bus system. 
The local manager is responsible for calculating the instantaneous location of the bus in the local area and estimating the site information. After the local manager performs the calculation, the bus data will be temporarily stored in the bus info recorder for local inquiry and bus info will be uploaded to the bus info database for permanent storage. When the connection to the central manager is broken, the local administrator can still provide operational services.

The display unit is responsible for displaying bus-to-station information, which can be an LCD/LED screen or a single-light information board.

Edge Layer: There are bus stops and bus vehicles in the edge layer. Bus vehicles include an onboard unit and a GPS system. Bus vehicles must transmit their location from time to time. Bus stops must periodically receive arrival information from stations on the route.

\subsection{Communication method}

In designing the transmission scheme, we mainly focused on the two key points of energy saving and low latency. The remainder of this section describes the communication method and transmission data format.

The current E-Bus information system [Fig. 13(a)] uses the mobile network (4G/LTE) to link buses and stop signs, and buses upload their current location to the data center. Every E-Bus stop sign shows the time the bus will arrive, which is updated every $1-10 \mathrm{~s}$. When the stop sign needs to obtain arrival time data, it initiates a request to the data center located in the cloud, and the data center returns the bus arrival time at the specific stop sign. This method has several issues in rural areas:

$\diamond$ The delay is long because most data centers are located in cities far from the countryside. In the process of data transmission, the number of nodes in the rural area is more than that in the city.

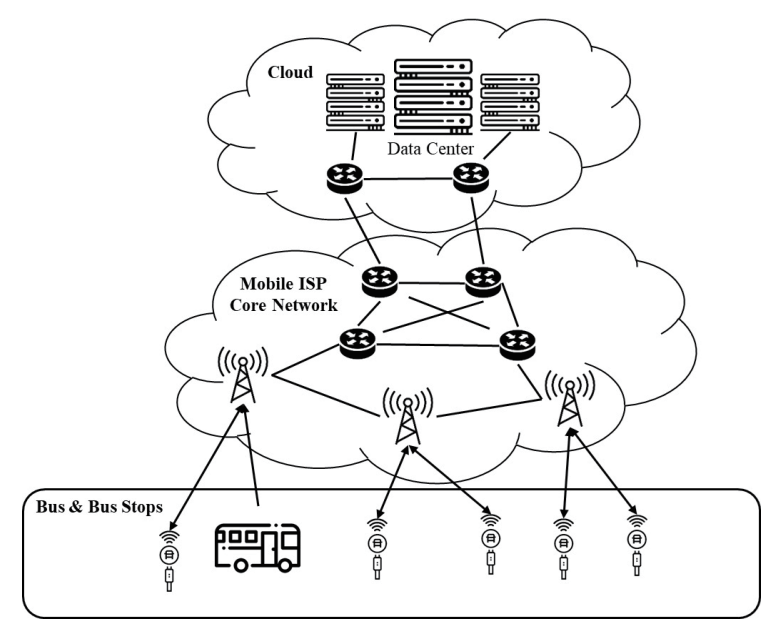

(a)

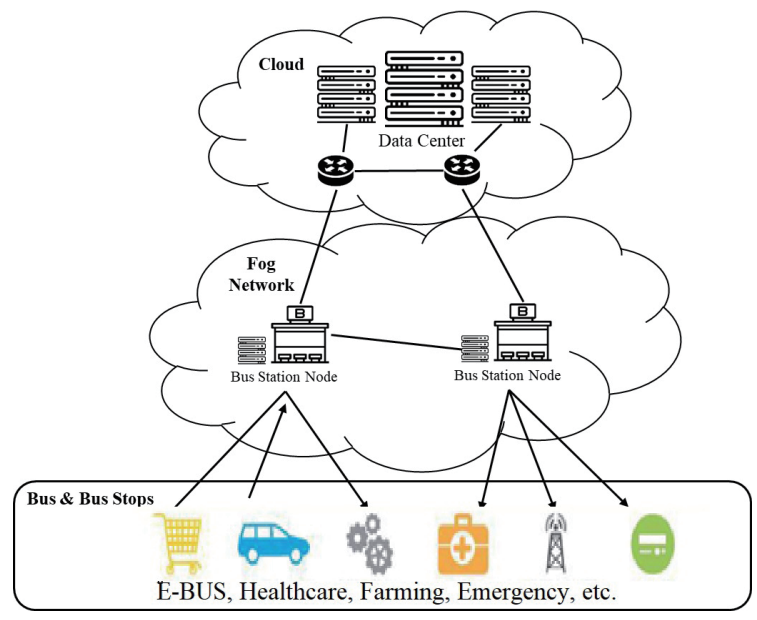

(b)

Fig. 13. (Color online) Communication method of (a) current E-Bus system and (b) rural E-Bus system. 
$\diamond$ The energy consumption is large, and the energy consumed by the mobile network is larger than that consumed by the LPWAN. When the base station signal is weak, the station card must consume more power to increase the transmission power.

In addition, because the connection between the station and the data center is Unicast, the number of stations correlates positively with the bandwidth consumption. This means that when the number of stations increases, the overall bandwidth consumption also increases.

Our transmission solution uses GloRa to link buses and stop signs [Fig. 13(b)]. GloRa's transmission distance and energy consumption performance are better than those of mobile networks. In addition, we will improve the way the station receives the bus arrival time. We will actively send the data by the bus stop node, the stop sign will passively receive the data, and the node will use the broadcast to transmit the data. This will greatly reduce the transmission delay and bandwidth usage due to the drastic reduction in the number of transmission nodes and transmissions.

\section{Conclusions}

In this paper, we propose a smart rural wide-area communication solution that is costeffective and energy-efficient. First, we introduce a low-latency localized smart fog-computing platform. In addition to solving rural mobile communication problems, it can also serve as an extension of the future IoT network. Furthermore, we use the E-Bus system as an example application and propose a low-latency, low-energy communication architecture and process. Finally, we set up a complete system in Jianshih Township, Hsinchu County. In addition to operating effectively, this system also provides a reliable local bus arrival forecasting service.

Next, we must connect the data among cities and rural areas by creating an IoT system through a new cloud and fog infrastructure. The integration of horizontal, vertical, and regional capabilities is critical when developing an IoT ecosystem. Technology providers may develop highly targeted, horizontal capabilities and integrate them with vertical and regional solutions.

In the future, we will live in a world with smart devices. The smart rural wide-area communication solution, in addition to positioning with the GPS information, can further explore whether a vehicle is driving illegally through cloud/fog calculation, thus achieving traffic safety monitoring. In the future, this system may be applied to other IoT applications, such as healthcare, disaster rescue, energy transformation, smart farming, travel arrangements, and environmental monitoring. Integrating cloud and fog computing will enable analysis, security, control, and data management at the edge of a network to be integrated with centralized cloud connectivity, policy, security, analytics, app development, and more. Through the combination of cloud computing and fog computing, the development of the IoT will be promoted and a new dawn of technological innovation will emerge. Improving the quality of life in rural areas is a joint responsibility of government, research, business, and society. Science and research should make every effort to support the pursuit of new and innovative paths. 


\section{Acknowledgments}

This work is supported by the Jianshih Township Office ITS project.

\section{References}

1 T. Boshita, H. Suzuki, and Y. Matsumoto: Proc. 2018 21st Int. Conf. Intelligent Transportation Systems (ITSC, 2018) 933-938. https://ieeexplore.ieee.org/document/8569920

2 Rural Communication: https://www.itu.int/en/ITU-D/Technology/Pages/RuralCommunications.aspx (accessed November 2018).

3 Outdoor Cycling Power: https://www.sunupeco.com/ (accessed October 2018).

4 J. Santa, R. Sanchez-Iborra, P. Rodriguez-Rey, L. Bernal-Escobedo, and A. F. Skarmeta: Sensors 19 (2019) 264. https://www.mdpi.com/1424-8220/19/2/264

5 E. Dahlman, G. Milch, S. Parkvall, J. Peisa, J. Sachs, Y. Selen, and J. Skold: IEEE Commun. Mag. December (2014) 42. https://ieeexplore.ieee.org/document/6979985

6 Enhancing Rural Transport Connectivity to Regional and International Transport Networks in Asia and the Pacific (ESCAP, Bangkok, 2019). https://www.unescap.org/sites/default/files/Monograph\%20on\%20Rural\%20 Transport $\% 20$ Connectivity $\% 20-\% 20 \mathrm{Full} \% 20$ text.pdf

7 D. Bruce: Survey of Rural and Regional Buses and On-demand Transport Services (ORIMA Research, Melbourne, 2017). https://www.ipart.nsw.gov.au/files/sharedassets/website/shared-files/pricing-reviewstransport-services-publications-rural-and-regional-bus-fares-2018-2022/orima-research-survey-of-rural-andregional-buses-and-on-demand-transport-services-october-2017.pdf

8 The-Future-of-Rural-Bus-Services (Tracks, UK, 2018). https://bettertransport.org.uk/sites/default/files/ research-files/The-Future-of-Rural-Bus-Services.pdf

9 E. H. K. Wu, J. Sahoo, C. Y. Liu, M. H. Jin, and S. H. Lin: IEEE Intell. Transp. Syst. Mag. 6 (2014) 35. https:// ieexplore.iee.org/document/6717140?arnumber $=6717140$

10 S. H. Lin, C. Y. Chen, E. H. K. Wu, C. Y. Chan, and E. Jou: IEEE Intell. Transp. Syst. Mag. 8 (2016) 51. https:// ieeexplore.ieee.org/document/7384611

11 L. Naldir, P. Nilsson, H. Westlund, and S. Wixe: J. Rural Stud. 40 (2015) 90. https://doi.org/10.1016/j. jrurstud.2015.06.006

12 E. H. K. Wu, C. Y. Chen, M. H. Jin, and S. H. Lin: Advanced Technologies, Embedded and Multimedia for Human-centric Computing (Springer, Dordrecht, 2013) p. 1247. https://doi.org/10.1007/978-94-007-7262-5_141

13 R. Oliveira, L. Guardalben, and S. Sargento: Proc. 2017 IEEE Symp. Computers and Communications (ISCC, 2017) 810-817. https://ieeexplore.iee.org/document/8024627

14 A. Augustin, J. Yi, T. Clausen, and W. M. Townsley: Sensors 16 (2016) 1466. https://pubmed.ncbi.nlm.nih. gov/27618064/

15 K-Best's IoT Tech Could Be Global Standard: http://www.taipeitimes.com/News/biz/ archives/2019/01/18/2003708116 (accessed October 2018).

16 Taiwan GloRa Alliance Established to Seize New AI and IoT Opportunities: https://mic.iii.org.tw/english/ AsiaExpress_Detail.aspx?doc_sqno $=12959 \&$ year $=2020 \& \mathrm{~m}=02 \&$ domain $\_$name $=\&$ domain $\_$sqno $=$(accessed October 2018).

17 LoRaWAN 1.1 Regional Parameters: https://iotas.ru/files/documents/LoRaWAN\%20Regional\%20 Parameters\%20v1.1rB.pdf (accessed November 2018).

18 H. Shah-Mansouri and V. W. S. Wong: IEEE Internet Things J. 5 (2018) 3246. https://ieeexplore.ieee.org/ document $/ 8360511$

19 T. H. Ashrafi, M. A. Hossain, S. E. Arefin, K. D. J. Das, and A. Chakrabarty: Proc. 2017 IEEE 3rd Int. Conf. Collaboration and Internet Computing (CIC, 2017) 163-172. https://ieeexplore.ieee.org/document/8181492 


\section{About the Authors}

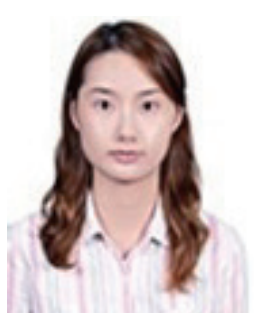

Shu-Hui Lin received an MBA degree from National Taiwan University of Science and Technology. She is currently a Ph.D. candidate at the Department of Computer Science and Information Engineering, National Central University. Her primary research interests include telematics, the Internet of Things, and radio technology. (littlemay_lin@yahoo.com.tw)

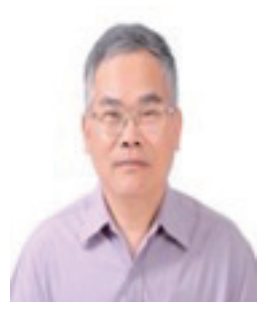

Yen-Hui Kuo received a master's degree in computer science from the University of Mississippi, USA. He is currently a Ph.D. student in the Department of Computer Science and Information Engineering at National Central University. He is also an advisory engineer at the Institute for Information Industry. His primary research interests include telematics, the Internet of Things, and wireless network technology and application. (kyh97730@gmail.com)

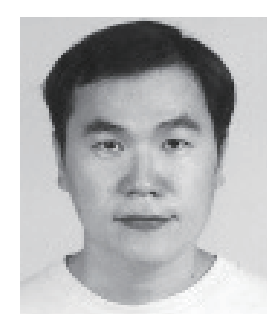

Eric Hsiao-Kuang Wu received his B.S. degree in computer science and information engineering from National Taiwan University in 1989. He received his master's and Ph.D. degrees in computer science from the University of California, Los Angeles (UCLA) in 1993 and 1997, respectively. $\mathrm{He}$ is a professor of computer science and information engineering at National Central University, Taiwan. His primary research interests include wireless networks, mobile computing, and broadband networks. He is a member of the Institute of Information and Computing Machinery (IICM) and IEEE. (hsiao@csie.ncu.edu.tw)

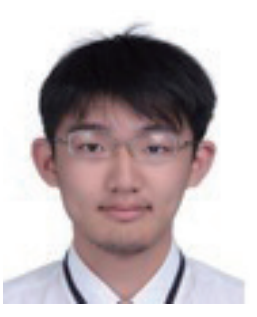

Hou-Sheng Lin received a B.S. degree in computer and communication engineering from Ming Chuan University, Taoyuan, Taiwan, in 2017. He is currently pursuing an M.S. degree in computer science and information engineering at National Central University, Taiwan. His research interests include the design and development of wireless communications, the Internet of Things, and locating technologies. (106522054@cc.ncu.edu.tw)

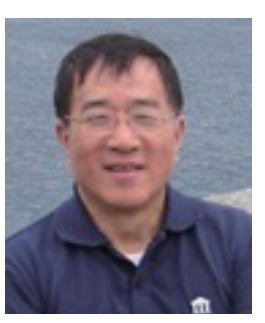

Emery Jou received his B.S. degree in physics from National Tsing Hua University, Hsinchu, Taiwan. He received his master's degree in computer science from the University of Texas, Austin, Texas. He received his Ph.D. degree in computer science from the University of Maryland, College Park, Maryland. He was a consultant at the Institute for Information Industry (III). His primary research interests include public key infrastructure, application performance testing, and B5G/6G IoT development. (emeryjou@gmail.com) 


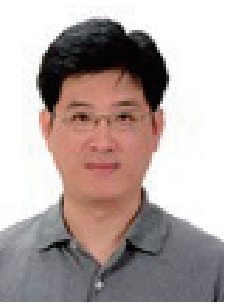

Ming-Hui Jin received his B.S. degree in mathematics from National Central University in 1995. He received his Ph.D. degree in computer science from National Central University in 2003. He is now an associate professor of the School of Internet Economics and Business at Fujian University of Technology. His primary research interests include game theory, healthcare, IoT, mobile computing, and modeling. (2476213978@qq.com) 